\title{
A Demonstration of Modern Bayesian Methods for Assessing System Reliability with Multilevel Data and for Allocating Resources
}

\author{
Todd L. Graves and Michael S. Hamada \\ Statistical Science, Los Alamos National Laboratory, Los Alamos, NM 87545, USA \\ Correspondence should be addressed to Todd L. Graves, tgraves@lanl.gov \\ Received 4 May 2009; Accepted 8 November 2009 \\ Recommended by Shuen-lin Jeng
}

Good estimates of the reliability of a system make use of test data and expert knowledge at all available levels. Furthermore, by integrating all these information sources, one can determine how best to allocate scarce testing resources to reduce uncertainty. Both of these goals are facilitated by modern Bayesian computational methods. We demonstrate these tools using examples that were previously solvable only through the use of ingenious approximations, and employ genetic algorithms to guide resource allocation.

Copyright (C) 2009 T. L. Graves and M. S. Hamada. This is an open access article distributed under the Creative Commons Attribution License, which permits unrestricted use, distribution, and reproduction in any medium, provided the original work is properly cited.

\section{Introduction}

Assessing the reliability of systems represented by reliability block diagrams remains important. Take for example, U.S. military weapon systems and nuclear power plants. In making these assessments, often there are information and data available at all levels of these systems, whether they be at the component, subsystem, or system level. For example, there may be data from component and subsystem tests as well as expensive full system tests. In this paper, we are concerned with assessing the reliability of a system by combining all available information and data at whatever level they are available; here we consider the case where we have success/failure test data.

Much of the reliability literature $([1-6])$ predates the advances made in Bayesian computation in the 1990s and resorts to various approximations. However, today a fully Bayesian method using the framework in [7], which simultaneously combines all available multilevel data and information, can be implemented using Markov chain Monte Carlo (MCMC). In this paper, we employ such modern Bayesian methods as MCMC to make reliability assessments.
In the next section, we introduce the statistical model that combines all available multilevel data and briefly present MCMC for analyzing such data. Then, we illustrate this methodology by making reliability assessments for an air-toair heat-seeking missile system and a low-pressure coolant injection system in a nuclear power plant first considered by $[5,6]$, respectively.

Once multilevel data and information can be analyzed, the question arises of what additional tests should be done when new funding becomes available. That is, what tests will reduce the system reliability uncertainty the most? In this paper, we show how a genetic algorithm using a preposteriorbased criterion can address this resource allocation question. Reference [8] considered resource allocation for a twocomponent series system. In this paper, we illustrate resource allocation with a more complex series-parallel system.

\section{A Model for Combining Multilevel Data}

To combine multilevel data for system reliability assessment, we use the framework in [7]. We introduce the framework's notation and models by considering the reliability block 
diagram of a series-parallel system given in Figure 1. First, components, subsystems, and the system are referred to as nodes. In this example, the system is node 0 which consists of two subsystems (nodes 1 and 2) in series. The first subsystem consists of two components in parallel (nodes 3 and 4) and the second subsystem consists of three components in series (nodes 5, 6, and 7).

We begin by considering the binomial data model when data are available at a node. At the $i$ th node, there are $x_{i}$ successes in $n_{i}$ trials with probability of success (reliability) $\pi_{i}$. If node $i$ is a subsystem or the full system (i.e., not a component), then $\pi_{i}$ is expressed in terms of the component reliabilities. For the series-parallel system, the subsystem reliabilities are expressed as $\pi_{1}=1-\left(1-\pi_{3}\right)\left(1-\pi_{4}\right)$ and $\pi_{2}=\pi_{5} \pi_{6} \pi_{7}$ and the system reliability is expressed as $\pi_{0}=\pi_{1} \pi_{2}=\left\{\left(1-\left(1-\pi_{3}\right)\left(1-\pi_{4}\right)\right) \pi_{5} \pi_{6} \pi_{7}\right\}$. In general, let $C$ be the subset of nodes which are components, and let $\pi_{C}=\left\{\pi_{i}: i \in C\right\}$; then for $i \notin C$ and for some function $h_{i}$, $\pi_{i}=h_{i}\left(\pi_{C}\right)$.

Next, we consider prior distributions for node reliabilities. For components, we use beta prior distributions in terms of an estimated reliability $\tilde{p}_{i}$ and a precision $v_{i}$ which acts like an effective sample size. That is, if the $i$ th node is a component, then $\pi_{i} \sim \operatorname{Beta}\left(\nu_{i} \tilde{p}_{i}, v_{i}\left(1-\tilde{p}_{i}\right)\right)$. If no information is available, the Jeffreys' prior $\operatorname{Beta}(1 \times 0.5,1 \times$ $(1-0.5))$ or a uniform prior $\operatorname{Beta}(2 \times 0.5,2 \times(1-0.5))$ can be used.

We also allow the possibility that information (expert knowledge) is available on the reliabilities of subsystems and/or the full system; we assume that this information is independent of the test data and any information used to build the prior distributions for the component reliabilities. (Frequently, we will not use any such information: in particular, expert opinion about upper-level nodes will often be based on the same information that led to the prior distributions for component reliabilities. This information should not be used twice, so a simple solution is to exclude the upper-level expert opinion.) Assume that the information takes the form of an estimated reliability $\tilde{p}_{i}$ and a precision $v_{i}$. We then express the information contribution, including the $x_{i}$ successful tests in $n_{i}$ trials, from the $i$ th subsystem or system as a term proportional to

$$
\pi_{i}^{x_{i}+\gamma_{i} \tilde{p}_{i}}\left(1-\pi_{i}\right)^{n_{i}-x_{i}+v_{i}\left(1-\tilde{p}_{i}\right)} .
$$

As discussed above, the subsystem or system reliability $\pi_{i}$ is expressed in terms of the component reliabilities as $h_{i}\left(\boldsymbol{\pi}_{C}\right)$. In effect, we have treated this information as if it were derived from binomial data instead of as a beta distribution; the difference involves a change in the exponents of $\pi_{i}$ and $\left(1-\pi_{i}\right)$ by one. One effect of this treatment is to ensure that the posterior distribution of $\pi_{C}$ is well defined. We can define $e_{i}$ to be the indicator that node $i$ is a component (i.e., $e_{i}=1$ if node $i$ is a component, and 0 , otherwise), in which case the information contribution from the $i$ th node is

$$
\pi_{i}^{x_{i}+v_{i} \tilde{p}_{i}-e_{i}}\left(1-\pi_{i}\right)^{n_{i}-x_{i}+v_{i}\left(1-\tilde{p}_{i}\right)-e_{i}},
$$

regardless of whether node $i$ is a component. If no information at the ith node is available beyond binomial tests, then
TABLE 1: Data for series-parallel system.

\begin{tabular}{lcc}
\hline Node & Data & $\tilde{p}$ \\
\hline 0 & $15 / 20$ & 0.8 \\
1 & & 0.9 \\
2 & $10 / 10$ & 0.9 \\
3 & $34 / 40$ & 0.9 \\
4 & $47 / 50$ & 0.9 \\
5 & $3 / 5$ & 0.95 \\
6 & $8 / 8$ & 0.95 \\
7 & $16 / 17$ & 0.95 \\
\hline
\end{tabular}

$v_{i}=0$, although $v_{i}>0$ should be used for components to ensure a proper prior. In the remainder of this paper, when we refer to the prior distribution, we mean the distribution that arises from combining the component Beta distributions with the upper-level expert knowledge. This is in fact a posterior distribution if there is nonzero expert knowledge, and in this case the components no longer have independent "prior" distributions.

A variety of models might be employed for the $v_{i}$. The $v_{i}$ might be treated as constants when they are really thought to be effective sample sizes. On the other hand, they might be described by a distribution, such as $\nu_{i} \sim \operatorname{Gamma}\left(a_{v}, b_{\nu}\right)$. This allows expert knowledge to be downweighted if it is inconsistent with the data. Now consider the data and prior information for the series-parallel system given in Table 1. Note that no precisions $v_{i}$ are provided so that a prior distribution needs to be specified. For illustration, we consider the same precision $v$, that is, $v_{i}=v$, and take the prior distribution for $v$ to be

$$
v \sim \operatorname{Gamma}\left(a_{v}=5, b_{v}=1\right) .
$$

That is, we believe that the expert information on average is worth five Bernoulli observations.

To combine the data with the expert knowledge represented as above, we use Bayes theorem

$$
f(\boldsymbol{\theta} \mid \mathbf{y})=\frac{f(\mathbf{y} \mid \boldsymbol{\theta}) f(\boldsymbol{\theta})}{\int f(\mathbf{y} \mid \boldsymbol{\xi}) f(\boldsymbol{\xi}) d \boldsymbol{\xi}},
$$

where $\boldsymbol{\theta}$ is the parameter vector (i.e., the component reliabilities $\boldsymbol{\pi}_{C}$ and any other unknown parameters), $\mathbf{y}$ is the data vector, $f(\boldsymbol{\theta})$ is the prior probability density function, and $f(\mathbf{y} \mid \boldsymbol{\theta})$ is the data probability density function (i.e., the binomial probability mass function for binomial data) which viewed as a function of the parameter vector given that the data is known as the likelihood. The result of combining the data with expert knowledge is $f(\boldsymbol{\theta} \mid \mathbf{y})$ which is known as the posterior distribution. Since the 1990s, advances in Bayesian computing through Markov chain Monte Carlo or MCMC have made it possible to sample from the posterior distribution [9]. Next, we discuss how the MetropolisHastings algorithm [10] can be used to obtain draws or samples from the parameter posterior distribution.

A fully Bayesian analysis of the model described above, which simultaneously combines all available multilevel data 


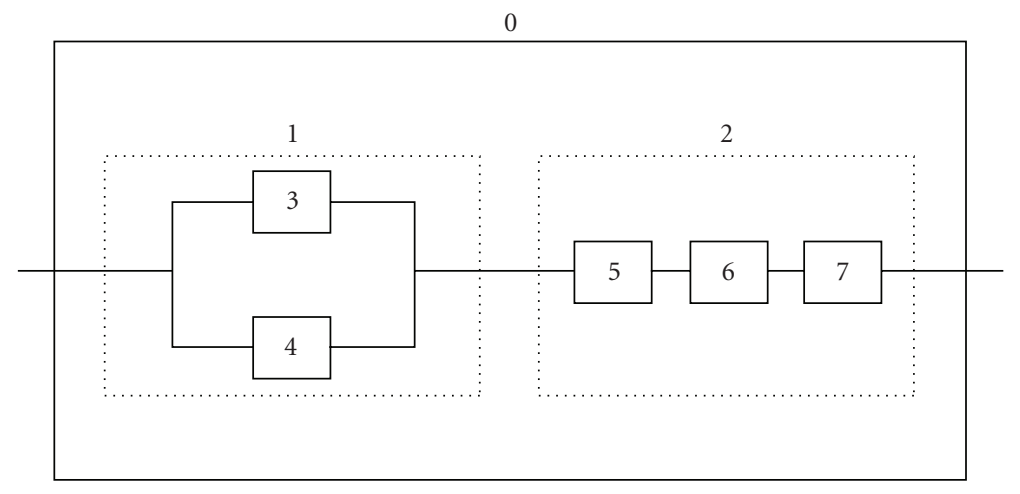

Figure 1: Series-parallel system reliability block diagram.

and information, is nontrivial. The posterior distribution is not analytically tractable: up to a normalizing constant, it is

$$
f\left(\boldsymbol{\pi}_{C}, \nu \mid \mathbf{x}, \tilde{\mathbf{p}}\right)=f_{\nu}(\nu) \prod_{i} \boldsymbol{\pi}_{i}^{x_{i}+\nu \tilde{p}_{i}-e_{i}}\left(1-\pi_{i}\right)^{n_{i}-x_{i}+\nu\left(1-\tilde{p}_{i}\right)-e_{i}}
$$

This looks superficially like a beta distribution, but it is not so simple because of the functional relationships between the $\pi_{i}$; that is, the subsystem and system $\pi_{i}=h_{i}\left(\pi_{C}\right)$. Consequently, a Bayesian analysis requires an implementation of an MCMC algorithm such as Metropolis-Hastings; see, for example, [10]. We use a variable-at-a-time MetropolisHastings algorithm as follows. The algorithm loops through all the unknown parameters $\pi_{i}(i \in C)$ and $\nu$, proposing changes to one parameter at a time and either accepting or rejecting changes according to the Metropolis-Hastings rule. We update the $\pi_{i}$ on the logit scale: suppose we are at the stage in one iteration of the algorithm where we are updating $\pi_{i}$ (for some $i \in C$ ). Propose a new value $\pi_{i}^{\prime}$ according to

$$
\operatorname{logit} \pi_{i}^{\prime} \sim \operatorname{Normal}\left(\operatorname{logit} \pi_{i}, s_{i}^{2}\right),
$$

where $s_{i}>0$ are tunable constants. Accept the value $\pi_{i}^{\prime}$ with probability

$$
\min \left\{1, \frac{\pi_{i}^{\prime}\left(1-\pi_{i}^{\prime}\right)}{\pi_{i}\left(1-\pi_{i}\right)} \frac{f\left(\pi_{C}^{\prime}, v \mid \mathbf{x}, \tilde{\mathbf{p}}\right)}{f\left(\boldsymbol{\pi}_{C}, v \mid \mathbf{x}, \widetilde{\mathbf{p}}\right)}\right\}
$$

where $\boldsymbol{\pi}_{C}^{\prime}$ is equal to $\boldsymbol{\pi}_{C}$ except with its $i$ th node reliability replaced by $\pi_{i}^{\prime}$. If the move is accepted, change the current value of the parameter to be $\pi_{i}^{\prime}$, otherwise its value continues to be $\pi_{i}$. After all the $\pi_{i}$ for $i \in C$ have been updated in this way, we update $v$ on the log scale; this proceeds similarly except that the proposed new values of $v$ satisfy

$$
\log \nu^{\prime} \sim \operatorname{Normal}\left(\log v, s_{\nu}^{2}\right)
$$

so that these proposed new values are accepted with probability

$$
\min \left\{1, \frac{v^{\prime}}{v} \frac{f\left(\boldsymbol{\pi}_{C}, v^{\prime} \mid \mathbf{x}, \tilde{\mathbf{p}}\right)}{f\left(\boldsymbol{\pi}_{C}, v \mid \mathbf{x}, \tilde{\mathbf{p}}\right)}\right\}
$$

After a complete iteration (after attempts to move each of the $\pi_{i}$ for $i \in C$ and also $\nu$ ), record the current values of all the parameters; this is treated as one sample from the posterior distribution. In practice the first several iterations are discarded as part of a "burn-in" period. Choosing good values of the $s_{i}$ is not difficult: in particular, the YADAS software system [11-13] has a method to tune these automatically in the burn-in period. This method consists of running an experiment with a wide range of $s_{i}$ 's, modeling the acceptance rates of the proposed moves using logistic regression with $\log \left(s_{i}\right)$ as a predictor, and choosing $s_{i}$ so that the logistic regression model predicts an acceptance rate close to a target value such as 0.35 .

The same MCMC algorithm just described for making draws from the joint posterior distribution can be used for making draws from the joint prior distribution

$$
f\left(\boldsymbol{\pi}_{C}, \nu \mid \tilde{\mathbf{p}}\right)=f_{\nu}(\nu) \prod_{i} \pi_{i}^{\nu \tilde{p}_{i}-e_{i}}\left(1-\pi_{i}\right)^{\nu\left(1-\tilde{p}_{i}\right)-e_{i}}
$$

where $\pi_{i}$ for a subsystem or system is a function of $\pi_{C}$. Draws for the subsystem and system reliabilities are obtained by evaluating the appropriate functions with the $\pi_{C}$ draws. The resulting prior distributions for the node reliabilities and $v$ are displayed as dashed lines in Figures 2 and 3, respectively.

In assessing the system reliability for the series-parallel system of Figure 1, we combine the node data with the prior distributions using MCMC as just described that result in the posterior distributions displayed as the solid lines in Figures 2 and 3. From these results, the 90\% (central) credible interval for the system (node 0), reliability is calculated as $(0.697,0.861)$ whose length is 0.164 . Note that even though there is no data for the first subsystem (node 1), the system data (node 0), and the component data (nodes 3 and 4), dramatically improve what we know about the first subsystem reliability. As shown in Figure 3, the addition of the data does not change $v$ much, except that $v$ is somewhat larger than indicated by the prior distribution; that is, the data essentially confirms the prior distribution of $\nu$. 


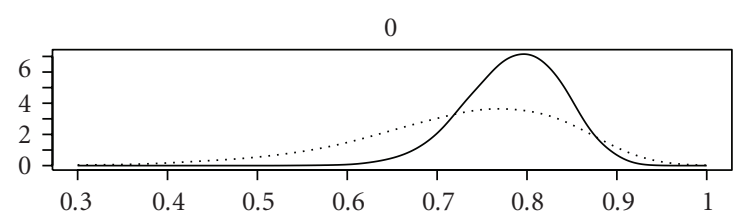

2
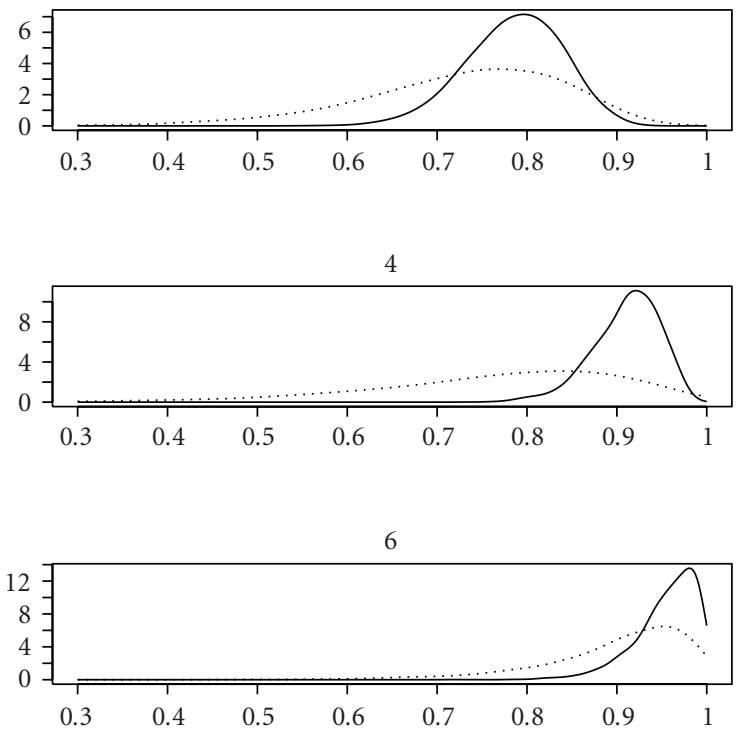

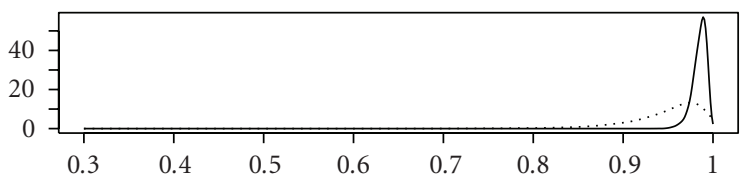

3

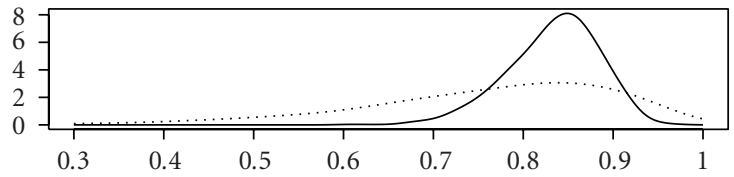

5

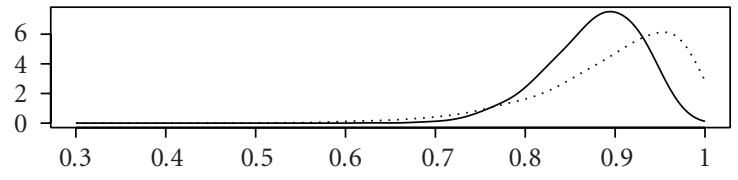

7

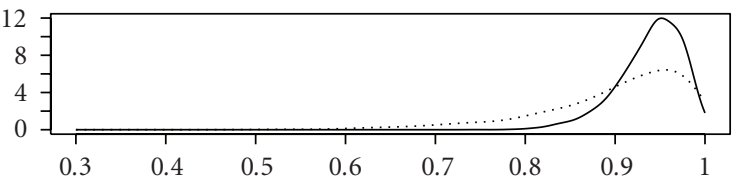

FIGURE 2: Plot of series-parallel system reliability priors (dashed lines) and posteriors (solid lines) for nodes 0-7.

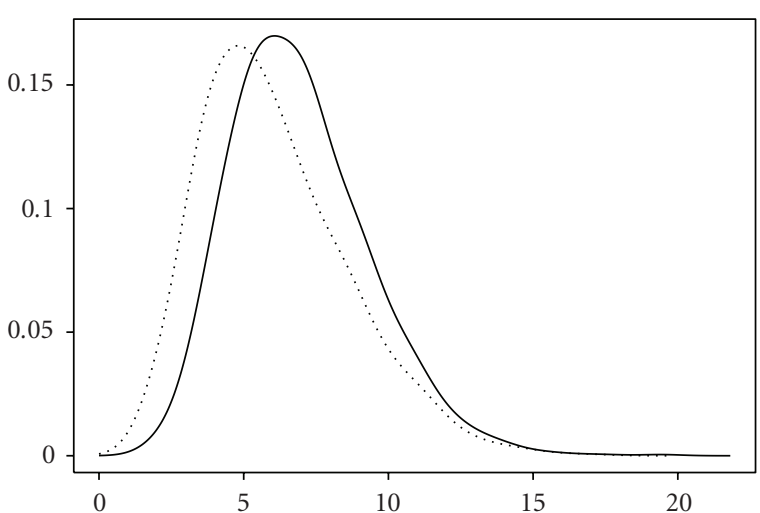

Figure 3: Plot of series-parallel system $v$ prior (dashed line) and posterior (solid line).

\section{Reliability Assessments for Two Applications}

Next, we consider two substantive applications from the literature $[5,6]$ to demonstrate making reliability assessments with multilevel data.

3.1. Series System Example. Reference [5] considered the reliability of a certain air-to-air heat-seeking missile system consisting of five subsystems in series each consisting of multiple components themselves combined in series as depicted in Figure 4. The data and prior information that [5] used are presented in Table 2 as (successes/trials) and estimated reliabilities $\tilde{p}$ and precisions $\nu$. Reference [5] did not provide details on how these data were obtained and how the prior information was arrived at.

To compare with [5], we treat the precisions as constants and then obtain the posterior node reliabilities using YADAS [11-13]. The posterior node reliabilities are displayed in Figure 5 as solid lines; the results from [5] are displayed as dashed lines. The median (0.50 quantile) and $90 \%$ credible intervals $(0.05,0.95$ quantiles) for the system and subsystem posterior reliabilities from the fully Bayesian and [5] methods are given in Table 3.

Note that there is quite a difference for the subsystem 1 results. The difference in location is due to the fact that the approximations used in [5] do not use higher-level information (system data) to estimate lower-level parameters (such as subsystem 1 reliability). The expert judgment estimate of system reliability, $116 / 267$ or 0.43 , is lower than the data and expert judgment at the lower levels would imply, and the fully Bayesian analysis needs to attribute this unreliability to one of the subsystems. Subsystem 1 and in particular component 19 has the sparsest information and is the natural targets. For this reason, the fully Bayesian analysis is more useful than the approach of [5] in evaluating the usefulness of gathering more data at low levels. In practice one would review the information that led to the low system reliability estimate. The fully Bayesian analysis could be rerun 


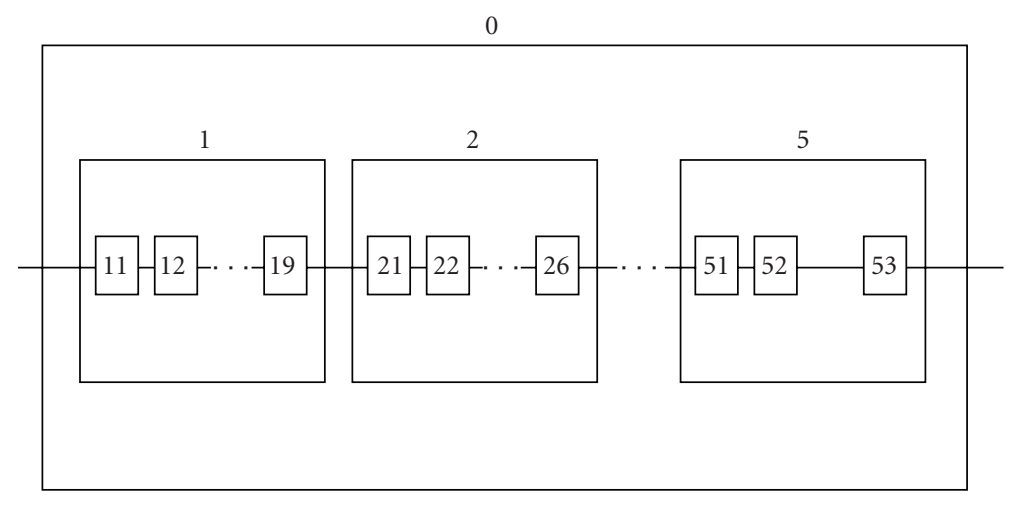

FIGURE 4: Series system example reliability block diagram.
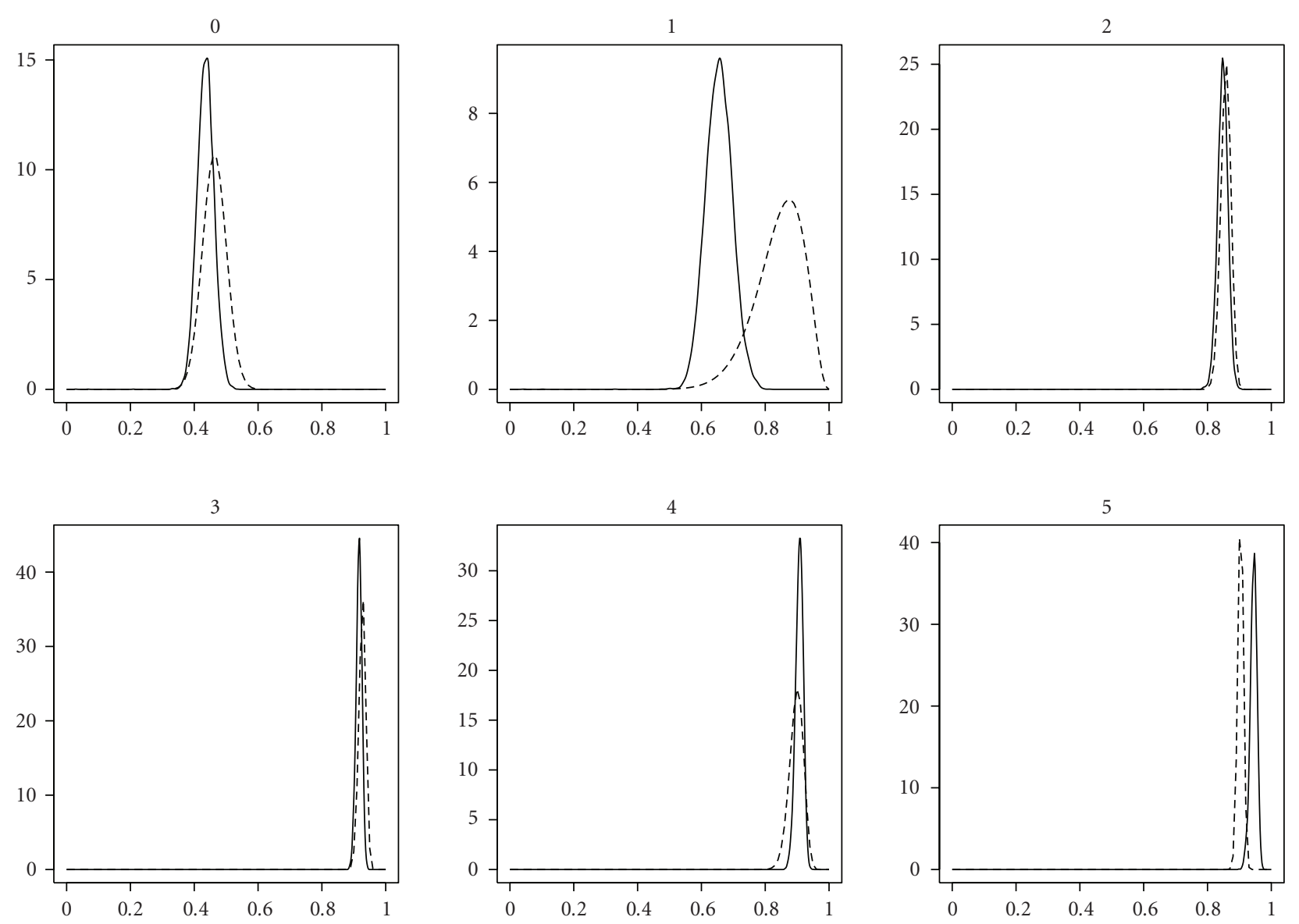

FIGURE 5: Plot of series system example reliability posteriors. (Dashed lines are from [5]. Solid lines are from fully Bayesian method.)

with random $\nu$ 's, and this would presumably allocate positive probability to the event that $\tilde{p}_{0}$ is an underestimate.

3.2. Complex Series-Parallel System Example. Reference [6] considered the reliability of a low-pressure coolant injection system, an important safety system in a nuclear-power boiling-water reactor. It consists of twin trains consisting of pumps, valves, heat exchanges, and piping whose reliability block diagram is displayed in Figure 6. The data and prior information that [6] used are presented in Table 4 as (successes/trials), estimated reliabilities $\tilde{p}$, and precision $\nu$.

Martz and Waller [6] based component prior distributions (i.e., for nodes 121, 122, 1111, 1112, 1121, 1122, 221, $222,2111,2112,2121,2122)$ on data from the Nuclear Regulatory Commission Accident Sequence Evaluation Program database [14] and some subsystem prior distributions (i.e., 
TABLE 2: Data for series system example.

\begin{tabular}{|c|c|c|c|}
\hline Node & Data & $\tilde{p}$ & $v$ \\
\hline 0 & & $115 / 265$ & 265 \\
\hline 1 & $8 / 8$ & & \\
\hline 2 & $7 / 8$ & & \\
\hline 3 & $191 / 205$ & $257 / 269$ & 269 \\
\hline 4 & & $55 / 66$ & 66 \\
\hline \multicolumn{4}{|l|}{5} \\
\hline 11 & $30 / 30$ & 0.5 & 1 \\
\hline 12 & $80 / 80$ & 0.5 & 1 \\
\hline 13 & $39 / 40$ & 0.5 & 1 \\
\hline 14 & $30 / 30$ & 0.5 & 1 \\
\hline 15 & $90 / 90$ & $846 / 848$ & 848 \\
\hline 16 & $10 / 10$ & 0.5 & 1 \\
\hline 17 & $29 / 30$ & 0.5 & 1 \\
\hline 18 & $20 / 20$ & 0.5 & 1 \\
\hline 19 & $5 / 5$ & 0.5 & 1 \\
\hline 21 & $50 / 50$ & $399 / 402$ & 402 \\
\hline 22 & $50 / 50$ & $278 / 302$ & 302 \\
\hline 23 & $99 / 100$ & $1098 / 1102$ & 1102 \\
\hline 24 & $23 / 25$ & $654 / 690$ & 690 \\
\hline 25 & $50 / 50$ & $299 / 301$ & 302 \\
\hline 26 & $55 / 55$ & $348 / 352$ & 352 \\
\hline 31 & $129 / 130$ & $246 / 250$ & 250 \\
\hline 32 & $130 / 130$ & $245 / 250$ & 250 \\
\hline 33 & $129 / 130$ & $247 / 250$ & 250 \\
\hline 34 & $129 / 130$ & $272 / 276$ & 276 \\
\hline 35 & $130 / 130$ & $357 / 360$ & 360 \\
\hline 36 & $247 / 250$ & $254 / 257$ & 257 \\
\hline 37 & $129 / 130$ & $250 / 252$ & 252 \\
\hline 38 & $249 / 250$ & $250 / 252$ & 252 \\
\hline 39 & $330 / 330$ & $341 / 352$ & 352 \\
\hline 41 & & $797 / 802$ & 802 \\
\hline 42 & & $796 / 802$ & 802 \\
\hline 43 & & $794 / 802$ & 802 \\
\hline 44 & & $791 / 802$ & 802 \\
\hline 45 & & $386 / 402$ & 402 \\
\hline 51 & & $1026 / 1122$ & 1122 \\
\hline 52 & & $1087 / 1092$ & 1092 \\
\hline 53 & & $1084 / 1092$ & 1092 \\
\hline
\end{tabular}

TABLE 3: Comparison of posteriors for series system example (0.05, $0.5,0.95$ quantiles).

\begin{tabular}{lcc}
\hline Node & Fully Bayesian & Reference [5] \\
\hline 0 & $(0.393,0.436,0.479)$ & $(0.403,0.463,0.525)$ \\
1 & $(0.588,0.655,0.723)$ & $(0.701,0.851,0.947)$ \\
2 & $(0.820,0.848,0.873)$ & $(0.830,0.858,0.883)$ \\
3 & $(0.901,0.917,0.931)$ & $(0.908,0.927,0.944)$ \\
4 & $(0.886,0.908,0.925)$ & $(0.858,0.898,0.931)$ \\
5 & $(0.926,0.945,0.961)$ & $(0.889,0.904,0.918)$ \\
\hline
\end{tabular}

TABLE 4: Data for complex series-parallel system example.

\begin{tabular}{|c|c|c|c|}
\hline Node & Data & $\tilde{p}$ & $v$ \\
\hline \multicolumn{4}{|l|}{0} \\
\hline \multicolumn{4}{|l|}{1} \\
\hline \multicolumn{4}{|l|}{2} \\
\hline \multicolumn{4}{|l|}{11} \\
\hline 12 & & $242.87 / 244.66$ & 244.66 \\
\hline 111 & & $1.55 / 1.58$ & 1.58 \\
\hline 112 & & $1.55 / 1.58$ & 1.58 \\
\hline 121 & $240 / 240$ & $470.13 / 471.90$ & 471.90 \\
\hline 122 & $240 / 240$ & $14232.34 / 14234.12$ & 14234.12 \\
\hline 1111 & $236 / 240$ & $191.17 / 191.79$ & 191.79 \\
\hline 1112 & $240 / 240$ & $14232.34 / 14234.12$ & 14234.12 \\
\hline 1121 & $238 / 240$ & $191.17 / 191.79$ & 191.79 \\
\hline 1122 & $240 / 240$ & $14232.34 / 14234.12$ & 14234.12 \\
\hline \multicolumn{4}{|l|}{21} \\
\hline 22 & & $242.87 / 244.66$ & 244.66 \\
\hline 211 & & $1.55 / 1.58$ & 1.58 \\
\hline 212 & & $1.55 / 1.58$ & 1.58 \\
\hline 221 & $240 / 240$ & $470.13 / 471.90$ & 471.90 \\
\hline 222 & $240 / 240$ & $14232.34 / 14234.12$ & 14234.12 \\
\hline 2111 & $240 / 240$ & $191.17 / 191.79$ & 191.79 \\
\hline 2112 & $240 / 240$ & $14232.34 / 14234.12$ & 14234.12 \\
\hline 2121 & $238 / 240$ & 191.17/191.79 & 191.79 \\
\hline 2122 & $240 / 240$ & $14232.34 / 14234.12$ & 14234.12 \\
\hline
\end{tabular}

for nodes $12,111,112,222,211,212)$ on composite IEEE Std. 500 reliability data ([15]). See [6] for more details.

We treat the precisions as constants as in [6], and then obtain the posterior node reliabilities using YADAS ([1113]). The resulting posterior reliabilities for the subsystems and system are displayed in Figure 7. Also, the summaries of the posterior reliabilities for all nodes are given in Table 5 . The results in Table 5 are similar to those given in [6] although somewhat smaller; for example, the $(0.05,0.5,0.95)$ quantiles for nodes 0-2 from [6] are $(0.999968,0.9999940$, $0.99999975),(0.9925,0.9974,0.99944)$, and $(0.9926,0.9974$, $0.99948)$, respectively.

\section{Resource Allocation}

In Section 2, we showed how to analyze multilevel data to assess system reliability. In this section we address test design. When additional funding becomes available, the question of where should the tests be done and how many should be taken arises to improve the system reliability assessment. In this section, we consider the optimal allocation of additional testing within a fixed budget that results in the least uncertainty of system reliability. We explore this by using the series-parallel system in Figure 1. We must determine how many tests should be performed at the system, subsystem, and component level (i.e., nodes 0-7) under a fixed budget for specified costs at each level (system, subsystem, component). In this paper, we use a genetic 


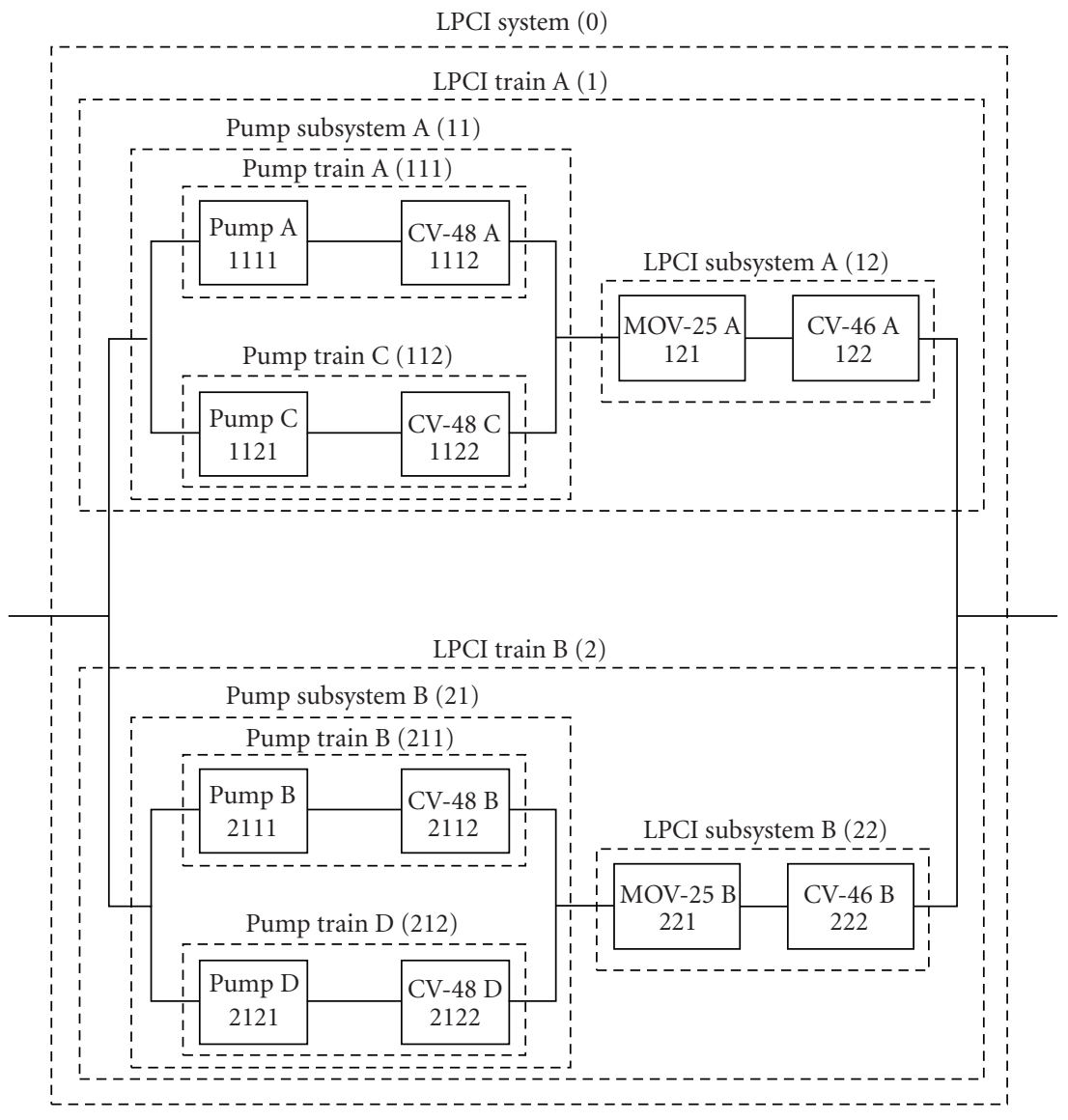

FIgURE 6: Complex series-parallel system example reliability block diagram.

algorithm (GA) $[16,17]$ to do the optimization because it is simple to implement and generally provides good results. But other optimization methods like particle swarms [18] could easily be used instead.

Thus, we assume that there is a cost for collecting additional data with higher-level data being more costly than lower-level data. Consider the following costs as an example of the costs for testing at each node. Recall that node 0 is the system, nodes 1 and 2 are subsystems and nodes 3-7 are components:

$$
\begin{aligned}
& (0: \$ 5), \quad(1: \$ 2), \quad(2: \$ 3), \quad(3: \$ 1), \quad(4: \$ 1), \\
& (5: \$ 1), \quad(6: \$ 1), \quad(7: \$ 1) \text {. }
\end{aligned}
$$

We evaluate a candidate allocation (i.e., a specified number of tests for each of the eight nodes) using a preposterior-based criterion as follows. We take a draw from the current joint posterior distribution (based on the current data) of the node reliabilities and draw binomial data according to the candidate allocation. Then we combine these new data with the current data using the same prior distributions to obtain an updated posterior distribution of the node reliabilities; again we use MCMC to obtain $N_{p}$ draws from this updated posterior distribution. The length of the $90 \%$ central credible interval of the system reliability posterior distribution is taken as a measure of uncertainty. This is repeated $N_{d}$ times, each with a different draw from the current joint posterior distribution of the node reliabilities. The uncertainty criterion is then calculated as the 0.90 quantile of the resulting $90 \%$ credible interval lengths.

Briefly, we describe how a GA can be used to find a nearly optimal allocation. A GA operates on a "population" of candidate allocations, where a candidate allocation is a vector of node test sizes. The GA begins by constructing an initial population or generation of $M$ allocations by randomly generating allocations that do not exceed the given fixed budget. The uncertainty criterion for each of these allocations in the initial population is evaluated and the allocations are ranked from smallest to largest, that is, the best allocation has the smallest criterion in the initial population. The second (and subsequent) GA generations are then populated using two genetic operations: crossover and mutation $[16,17]$. A crossover is achieved by randomly selecting two parent allocations from the initial (or current) generation without replacement with probabilities inversely proportional to their rank among the $M$ allocations in the initial (or current) generation. A new allocation is generated node by node from these two selected parent allocations by randomly picking one of the two parents each time and taking its node test size. The two parent allocations are then returned to the initial (or current) population before the 

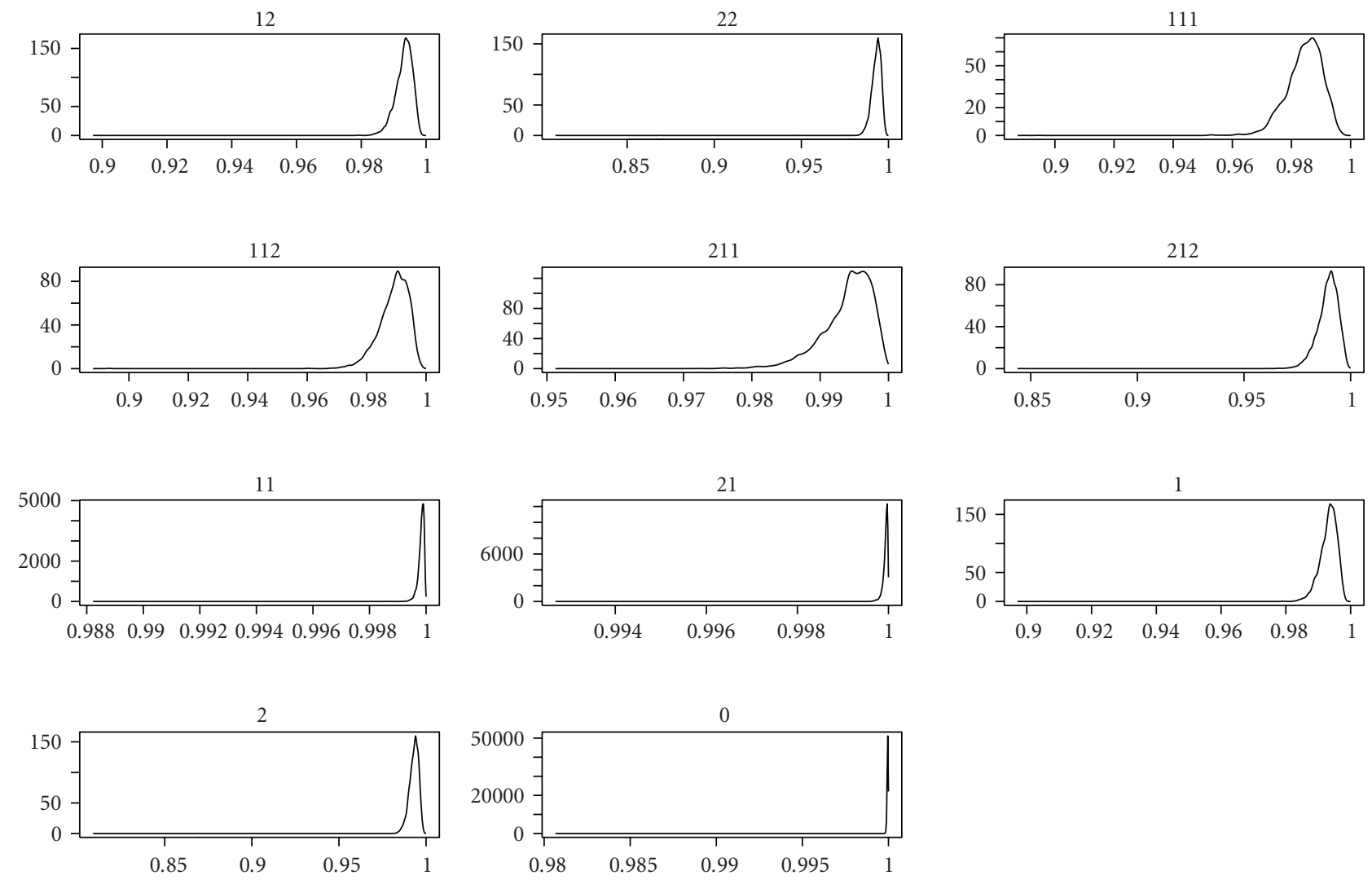

FIGURE 7: Plot of complex series-parallel system example reliability posteriors for subsystems and system.

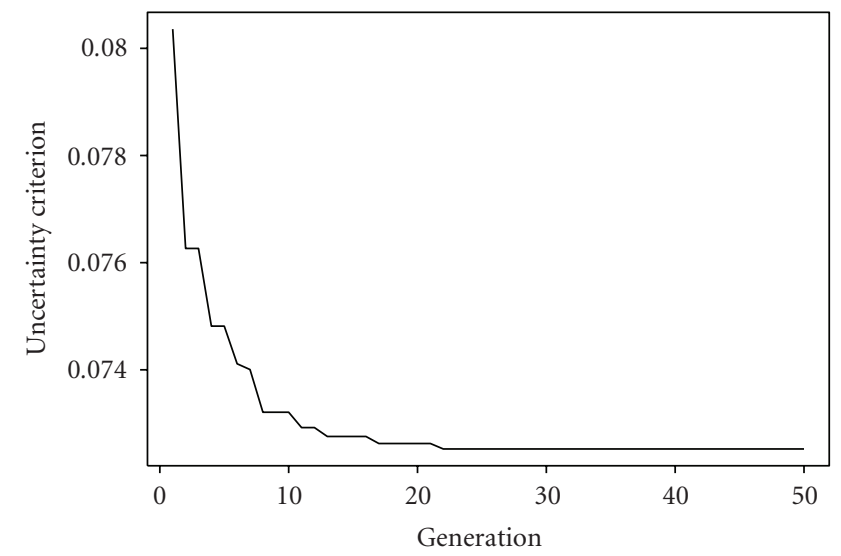

FigURE 8: GA evolution of uncertainty criterion.

next crossover is performed. In this way, an additional $M$ allocations are generated using the crossover operator. The generated allocations are checked to make sure they do not exceed the budget, so that new allocations are generated until there are $M$ such allocations. The uncertainty criterion is then evaluated for each of these new allocations. A mutation of each of the initial (or current) $M$ allocations is obtained node by node by first randomly deciding to change the node test size and if so then randomly perturbing the current node

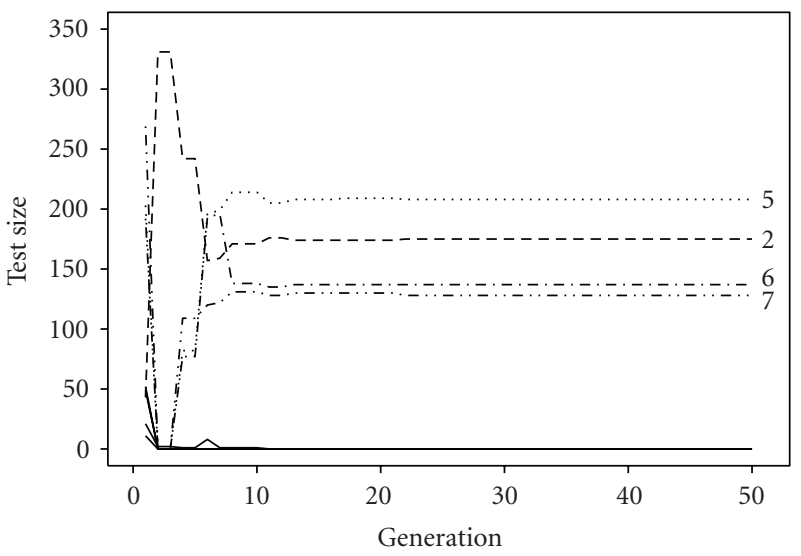

Figure 9: GA evolution of resource allocation. Nodes 2 and 5-7 test sizes are identified.

test size. Using mutation, $M$ additional allocations which remain within the budget are generated and the uncertainty criterion for each is evaluated. At this point there are $3 \mathrm{M}$ allocations. In the next generation, the current population consists of the $M$ best allocations from these $3 M$ allocations, that is, with the smallest uncertainty criterion. The GA is executed for $G$ generations. We implemented the GA for resource allocation in $R$ [19] which generates the candidate allocations. An allocation is evaluated in $R$ by repeatedly 
TABle 5: Posterior summaries for complex series-parallel system example (0.05, 0.5, 0.95 quantiles).

\begin{tabular}{ll}
\hline Node & $(0.99990,0.99996,0.99998)$ \\
\hline 0 & $(0.98835,0.99354,0.99678)$ \\
1 & $(0.98812,0.99342,0.99680)$ \\
11 & $(0.99962,0.99985,0.99995)$ \\
12 & $(0.98835,0.99354,0.99678)$ \\
111 & $(0.97371,0.98505,0.99311)$ \\
112 & $(0.97990,0.98990,0.99568)$ \\
121 & $(0.98853,0.99373,0.99696)$ \\
122 & $(0.99962,0.99985,0.99996)$ \\
1111 & $(0.97389,0.98519,0.99329)$ \\
1112 & $(0.99962,0.99986,0.99996)$ \\
1121 & $(0.98010,0.99008,0.99584)$ \\
1122 & $(0.99961,0.99985,0.99996)$ \\
21 & $(0.99983,0.99995,0.99999)$ \\
22 & $(0.98812,0.99342,0.99680)$ \\
211 & $(0.98681,0.99468,0.99847)$ \\
212 & $(0.98040,0.98994,0.99599)$ \\
221 & $(0.98826,0.99359,0.99697)$ \\
222 & $(0.99962,0.99985,0.99996)$ \\
2111 & $(0.98697,0.99485,0.99865)$ \\
2112 & $(0.99961,0.99986,0.99996)$ \\
2122 & $(0.98054,0.99011,0.99614)$ \\
& $(0.99962,0.99985,0.99996)$ \\
\hline
\end{tabular}

building YADAS [11-13] input data files, running the YADAS code using the reliability package (through the $R$ "system" call) to analyze the new and current data, and reading the resulting YADAS output files back into $R$ to calculate the uncertainty criterion.

In the implementation, there are a number of issues regarding the choice of $M, G, N_{p}$, and $N_{d}$. As the population size $M$ and number of generations $G$ increase, more candidate allocations (i.e., $M(1+2 \times G))$ are entertained, but then more calculation is required. As the number of posterior draws for each generated data set $N_{p}$ and the number of generated data sets to analyze $N_{d}$ increase, the uncertainty criterion is better evaluated, but the calculation needed to evaluate a single candidate allocation can dramatically increase let alone that for $M(1+2 \times G)$ candidate allocations. One has to realize that the nearly optimal allocation found by the GA may not be the optimal allocation if the difference between them is less that the variability of the evaluated uncertainty criterion, that is, within the simulation error of the uncertainty criterion.

One might ask if there are any general insights regarding resource allocation with assessment of system reliability in mind. If we consider testing at the same level, for components (or subsystem), the component (or subsystem) with the most uncertainty will require more testing than the others. If the subsystems are connected in series, but some subsystems have components connected in series where as other subsystems have components connected in parallel, in terms of component testing, the parallel configured subsystems will require less testing; this can be explained by examining the subsystem reliability expression, which shows that the reliability of series configured subsystems is of second order in their component reliabilities, where as that for parallel configured subsystems is of first order. The allocation will also depend on the testing costs relative to the amount of uncertainty reduction that it provides. If we consider a series configured subsystem, if the subsystem cost exceeds the sum of the components costs, then performing components tests will be recommended; if the subsystem cost is less than the sum of the components costs, then performing some subsystem tests may be recommended if they provide relatively more information. But for complicated systems with many subsystems and components whose costs are all different, it will be difficult to choose an optimal allocation with these rules of thumb. However, the proposed methodology balances all these costs and information across the entire system in finding a nearly optimal allocation.

Next, we illustrate the GA for the resource allocation problem described above for the series-parallel system depicted in Figure 1 for a fixed budget of $\$ 1000$. The length of the $90 \%$ credible interval of system reliability based on the existing data is 0.164 . We use populations of size $M=20$ and $G=50$ generations, so that $2020(=20(1+2 \times 50))$ candidate allocations were generated and evaluated. To evaluate the uncertainty criterion, we generated $N_{p}=2000$ posterior draws per data analysis and generated $N_{d}=500$ data sets corresponding to posterior draws based on the existing data. For this situation, what allocation yields the most reduction in the uncertainty criterion for system reliability?

Based on the proposed methodology described above, the GA produced the traces presented in Figures 8 and 9 which display the best uncertainty criterion and allocation found during each generation. The uncertainty criterion drops to 0.0804 for the initial population and decreases to 0.0725 by generation 50 with an allocation of test sizes $(0$, $0,175,0,0,208,137,128)$ for nodes $0-7$. We evaluated this allocation with $N_{p}=50000$ and $N_{d}=100000$ and obtained uncertainty criterion values of 0.073358 and 0.073363 , so we take the uncertainty criterion for this allocation as 0.0734 . These results suggest that there is enough data for node 1 , the two component parallel subsystems and the cost structure prohibit additional system tests (i.e., the system cost equals the sum of the subsystem costs, which equals the sum of the components costs). Because node 2 subsystem cost equals the sum of its component costs, we tried an allocation which proportionally allocated the subsystem tests to its components (i.e., splitting up $175 \times 3=525$ by the proportion $(208 / 473,137 / 473,128 / 473)$ found by the GA) giving the allocation $(0,0,0,0,0,439,289,270)$. Evaluating this allocation again with $N_{p}=50000$ and $N_{d}=$ 100000 gave uncertainty criterion values of 0.071439 and 0.071426 , which we round to 0.0714 . Consequently, there is some improvement by doing all component tests for node 2 subsystem. 


\section{Discussion}

For relatively complex systems, we have illustrated how to respond to the challenge of integrating all information available at the various levels of a system in order to estimate its reliability. Bayesian models have always been natural for doing this integration, and the computational tools have now caught up to make this practical. Moreover, because we are able to analyze such data, we can now consider the problem of allocating additional resources that best reduce the uncertainty in the system reliability assessment.

We have discussed the case of binomial test data only for systems represented by reliability block diagrams. Reference [20] showed how binomial data can be analyzed for problems using fault tree representations. Component and subsystem tests may generate continuous data such as lifetimes, and their distributions may depend on covariates such as different suppliers. Reference [21] presented an example of such an analysis. However, the problem of resource allocation for nonbinomial test data is a topic for future research.

\section{Acknowledgments}

The authors thank C. C. Essix for her encouragement of this work and Vivian Romero for her assistance in producing the reliability block diagram figures used in this paper. We also thank the referees for helpful comments that improved the presentation of this paper.

\section{References}

[1] P. V.Z. Cole, "A Bayesian reliability assessment of complex systems for binomial sampling," IEEE Transactions on Reliability, vol. 24, no. 2, pp. 114-117, 1975.

[2] D. V. Mastran, "Incorporating component and system test data into the same assessment: a Bayesian approach," Operations Research, vol. 24, no. 3, pp. 491-499, 1976.

[3] D. V. Mastran and N. D. Singpurwalla, "A Bayesian estimation of the reliability of coherent structures," Operations Research, vol. 26, no. 4, pp. 663-672, 1978.

[4] B. Natvig and H. Eide, "Bayesian estimation of system reliability," Scandinavian Journal of Statistics, vol. 14, no. 4, pp. 319-327, 1987.

[5] H. F. Martz, R. A. Walter, and E. T. Fickas, "Bayesian reliability analysis of series systems of binomial subsystems and components," Technometrics, vol. 30, no. 2, pp. 143-154, 1988.

[6] H. F. Martz and R. A. Waller, "Bayesian reliability analysis of complex series/parallel systems of binomial subsystems and components," Technometrics, vol. 32, no. 4, pp. 407-416, 1990.

[7] V. E. Johnson, T. L. Graves, M. S. Hamada, and C. S. Reese, "A hierarchical model for estimating the reliability of complex systems," in Bayesian Statistics 7, J. M. Bernardo, M. J. Bayarri, J. Berger, et al., Eds., pp. 199-213, Oxford University Press, London, UK, 2003.

[8] A. G. Wilson, T. L. Graves, M. S. Hamada, and C. S. Reese, "Advances in data combination, analysis and collection for system reliability assessment," Statistical Science, vol. 21, no. 4, pp. 514-531, 2006.
[9] A. E. Gelfand and A. F. M. Smith, "Sampling-based approaches to calculating marginal densities," Journal of the American Statistical Association, vol. 85, no. 410, pp. 398-409, 1990.

[10] S. Chib and E. Greenberg, "Understanding the MetropolisHastings algorithm," The American Statistician, vol. 49, pp. 327-335, 1995.

[11] T. L. Graves, "An Introduction to YADAS," 2003, http://www .stat.lanl.gov/yadas/yadas.pdf.

[12] T. L. Graves, "The YADAS reliability package," Tech. Rep. LA-UR-06-7739, Los Alamos National Laboratory, Los Alamos, NM, USA, 2006, http://wwwstat.lanl.gov/yadas/ node1.html\#download.

[13] T. L. Graves, "Design ideas for Markov chain Monte Carlo software," Journal of Computational and Graphical Statistics, vol. 16, no. 1, pp. 24-43, 2007.

[14] U.S. Nuclear Regulatory Commission, "Reactor risk reference document (vols. 1-3, draft)," Tech. Rep. NUREG-1150, 1987.

[15] Institute of Electrical and Electronic Engineers, IEEE Guide to the Collection and Presentation of Electrical, Electronic, Sensing Component, and Mechanical Equipment Reliability Data for Nuclear-Power Generating Stations, Wiley-Interscience, New York, NY, USA, 1983.

[16] D. E. Goldberg, Genetic Algorithms in Search, Optimization and Machine Learning, Addison-Wesley, New York, NY, USA, 1989.

[17] Z. Michalewicz, Genetic Algorithms + Data Structures = Evolution Programs, Springer, New York, NY, USA, 1992.

[18] R. C. Eberhart and J. Kennedy, "A new optimizer using particle swarm theory," in Proceedings of the 6th International Symposium on Micro Machine and Human Science, pp. 39-43, IEEE Service Center, Nagoya, Japan, October 1995.

[19] R Development Core Team, R: A Language and Environment for Statistical Computing, R Foundation for Statistical Computing, Vienna, Austria, 2004, http://www.R-project.org.

[20] M. Hamada, H. F. Martz, C. S. Reese, T. Graves, V. Johnson, and A. G. Wilson, "A fully Bayesian approach for combining multilevel failure information in fault tree quantification and optimal follow-on resource allocation," Reliability Engineering and System Safety, vol. 86, no. 3, pp. 297-305, 2004.

[21] T. L. Graves and M. S. Hamada, "Bayesian methods for assessing system reliability: models and computation," in Modern Statistical and Mathematical Methods in Reliability, A. Wilson, N. Limnios, S. Keller-McNulty, and Y. Armijo, Eds., vol. 10 of Series on Quality, Reliability, and Engineering Statistics, pp. 41-54, World Scientific, Singapore, 2005. 

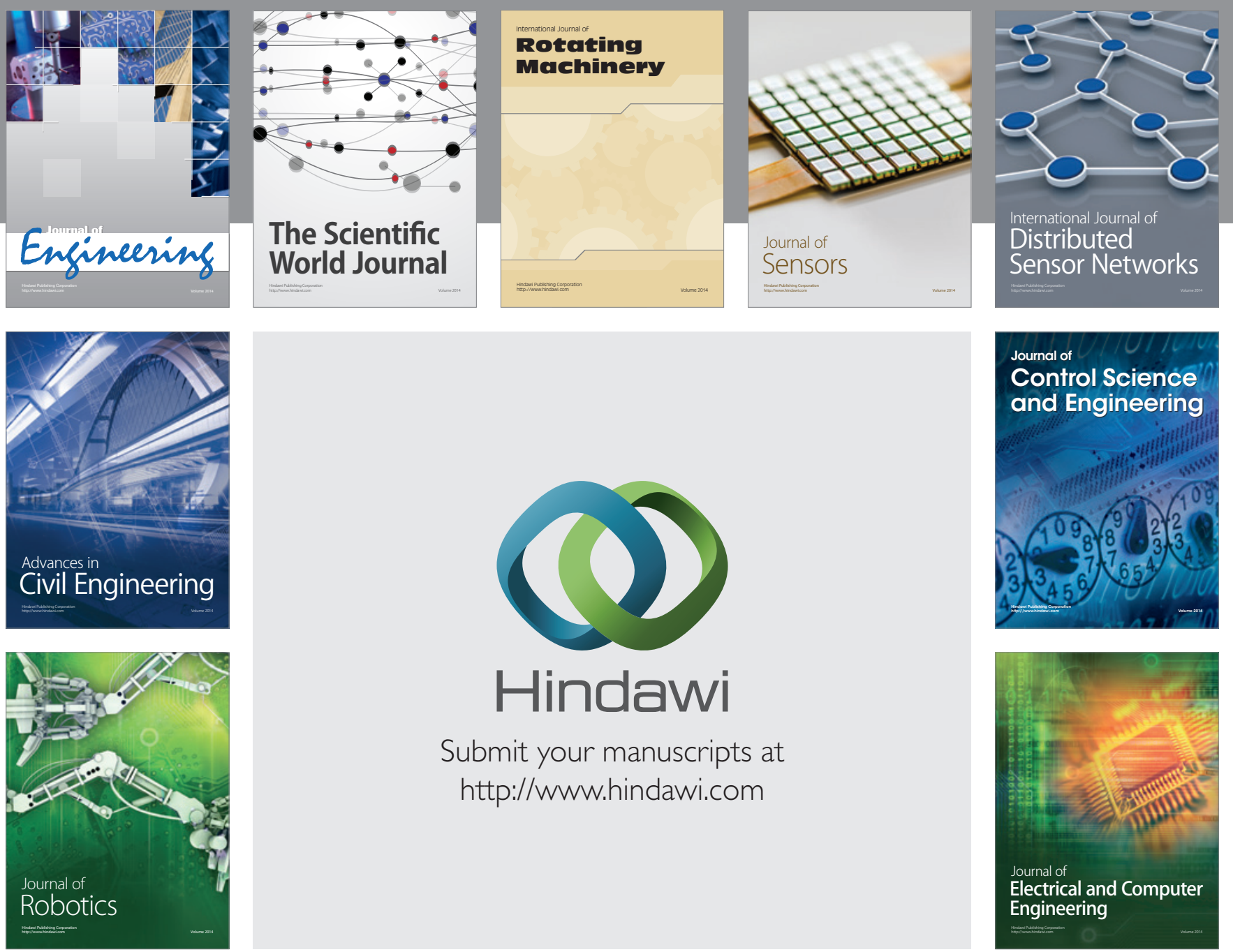

Submit your manuscripts at

http://www.hindawi.com
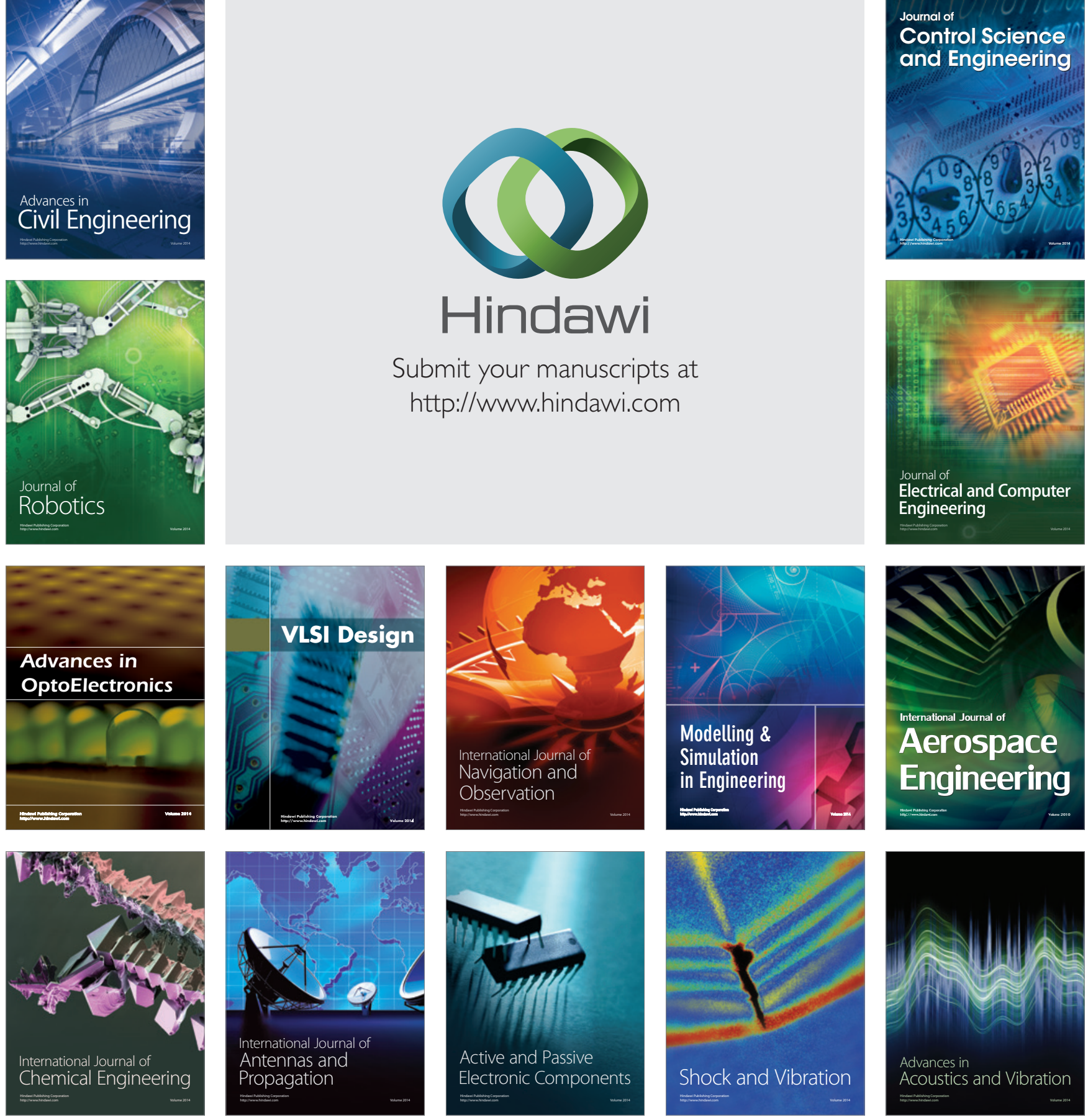